\title{
Innovative Solutions of the Automated Guided Vehicles in Industrial Manufacturing
}

\author{
Dan Bizubac ${ }^{1, *}$, Bernd Otto Hörmann ${ }^{1}$ and Marcel Sabin Popa ${ }^{1}$ \\ ${ }^{1}$ Technical University of Cluj-Napoca, Department of Manufacturing Engineering, Muncii Blvd 103- \\ 105, Cluj-Napoca, ROMANIA
}

\begin{abstract}
The Automated Guided Vehicle (AGV) is a mobile device that is used in the manufacturing plants lately for transporting materials from one space to another. AGVs are connected to a central navigation system which continuously directs the device to its source or destination. Their main features are their flexibility and adaptability to the environment once the AGVs are configured. The main focus of this article is to present the AGV technology based on vision navigation system reinforced by programming code and navigation graphs with the scope of adhering to the latest innovative concepts in terms of efficiency and optimized manufacturing. The topic of vision-based systems being recent to the engineering evolution, several academicians have discussed probabilities for improvement: some introduce the concept of an independent robot which can learn through artificial intelligence about the dynamic environment or others state, that a neural network can be used in the future as the basis for movement of the AGV in the workspace.
\end{abstract}

\section{Literature Review}

The first step in developing an automated guided vehicle was taken by Barnet [1] in 1953 when he engineered a vehicle controlled by wires in order to support in navigating through the aisles of a supermarket. Since this experiment, the modern technology of AGVs has developed and has improved significantly by assisting also other engineering fields. In 2013, Punithe [1] has brought the AGV technology in the medical system in order to improve the processes within. The AGV technology has been used since its initial development in other fields such as: hotel industry, warehousing or other robot mechanized departments of a manufacturing industry. In this direction, Feng [2] states that once the AGV technology is sustainable and fully developed, the vehicles will be able to reimburse their initial financial costs and labour time for configuration.

The vehicles that are the core for this technology are nearly automated transportation programmable mobile utilities. These have been lately introduced to the manufacturing industry where transportation is being done with low expenses after the system has been implemented according to a specific and configured route. The route can be configured using different methods such as selection of a frequency mode, path mode or even through vision modes.

\footnotetext{
* Corresponding author: danbizubac@yahoo.com
} 
Depending on the type of system implemented, there are two different scenarios. On one hand, there can be a core system which issues every time when needed a speed and steering command or on the other hand, a different system is represented by the AGV technology that stores the map of the facility in its internal physical memory. The AGV complete system contains the following components: the actual vehicles, the core-control management system and the guidance/path system. It has been demonstrated by academics [3] that the efficiency of an AGV system is performant when there is a faultless connection between all the above items.
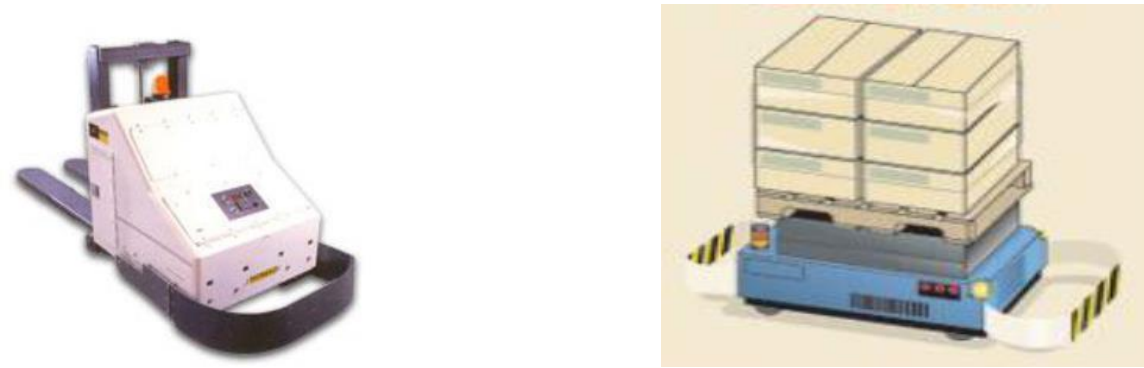

Fig. 1. Left - Transporters for the carried load on a predetermined path; Right - AGV transporters for the carried load from manufacturing stations to other stations.

As already mentioned by the academic literature [3], the AGV system is as powerful as it is well-controlled by the predetermined routes. These predetermined routes are being developed in the initial configuration of the system using programming paths. A normal movement in the $\mathrm{AGV}$ is the regular transportation on a linear path from point $\mathrm{A}$ to point $\mathrm{B}$. In this case, sensors are used, and they are constantly compared to the existing values in the configuration. In the decision points, the AGV reassesses the information captured by the sensors (in the live environment) and it adjusts its movements to the predefined values in order to turn left or right, as needed by the movement graph [3].

In practice, three different type of guidance systems have evolved: landmark navigation, behaviours navigation and vision navigation. Landmark navigation is based on the recognition of the special environment features of the existing manufacturing space that have been previously uploaded in the system. This comparison is usually implemented using different taped type system or embedded guided wires. In the case of the embedded system, different wires are inserted in the ground floor of the working space. Based on these, the AGV senses the frequency of $1-15 \mathrm{kHz}$ and controls the motor and steering in order to follow the desired direction. On the other hand, the guided tape uses a predefined marked line on the floor as a guiding system. In order to remain on the designated pathway, the robot uses a sensor which send bidirectional a signal to the core system with its latest finding. Rahaman [4] implements in his work the AGV using a servo motor guided by an infrared three sensor system linked to a PIC-16F877A microcontroller in order to avoid accidents with other environmental objects. From a different perspective, Bajestani [5] used a follower device as a robot and an ATMEGA 16 microcontroller. It is managed in this way, how to make the robot turn 90 degrees and thus improving the robot capabilities and performance. Also, by using LDR and LED systems for navigation, the robot's performance was not depending anymore on the light exposure from the manufacturing area.

The second type of navigation, the behaviour system was demonstrated to be effective in unstructured environments with many sensors. For a successful use, it required a state-ofthe-art network, power and a well-defined algorithm. From a physical point of view, laser recognition is being used for speed and steering. In his work, Kelly [6] has discussed about 
using this strategy in order to improve the precision of the AGV. Nevertheless, the technology has been assessed as having a high-cost and depended on the reflection marker.

Vision Based System have been the latest technology in the AGV manufacturing industry. This technology uses the navigation system in order to determine its position in an autonomous way. This AGV hybrid uses in its navigation a minimum required sensor system which captures the proximity to different objects through visual images. The topic of vision-based systems being recent to the engineering evolution, several academicians have discussed probabilities for improvement: Rajiv [7] introduced the concept of an independent robot which can learn through artificial intelligence about the dynamic environment or Kishor stated that a neural network can be used in the future as the basis for movement of the AGV in the workspace.

\section{Methodology}

The methodology used by the author in the implementation is described using the product from SAP: Plant Connectivity and Manufacturing Integration and Intelligence. SAP Plant Connectivity (PCo) is used as in intermediate data exchanger between the physical level represented by the AGV technology and the upper programming level on the architecture represented by Manufacturing Integration and Intelligence (MII). The AGV technology used at the lower level of coding decision has been chosen to be the vision based navigation system combined with a hybrid pathway uploaded in the AGV's internal memory.

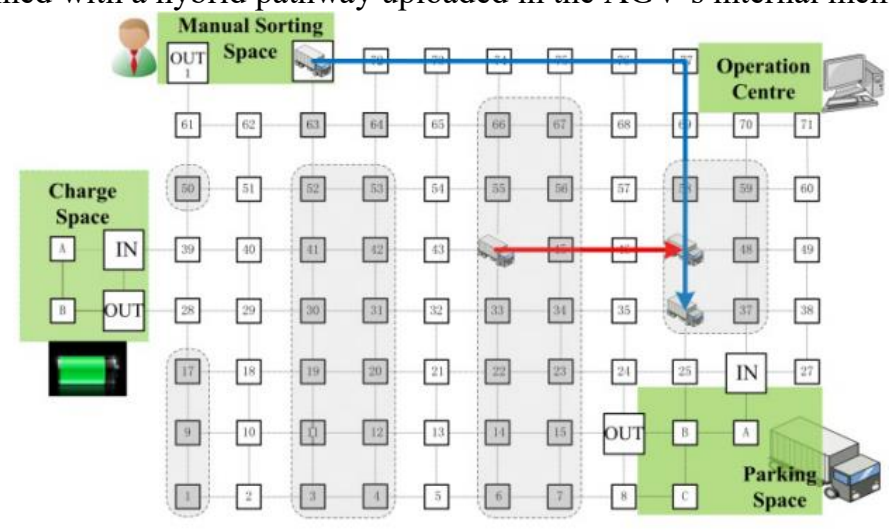

Fig. 2. Example of the predetermined routes/pathway for the AGV.

After careful evaluation of a predetermined testing space, the authors have recreated for academic testing, the environment of a manufacturing plant as in Fig 2.

In terms of the logic developed within, the AGVs were uploaded with the set of navigation routes in the second stage of the academic experiment. Meanwhile, in the decision-making levels such as PCo, the authors have implemented a buffer system which connect the AGV to the advanced level of coding. The buffer stores bidirectional the information for the AGV for a better connection and to avoid data losses. At the MII level, a coding webservice CallPcoWebService has been implemented, in order to collect the data bidirectionally from the stations and to send it towards the AGVs. Now, the decision is made wherever to call a new AGV to transport the material to the next station if it is finished in the current station or to bring raw material for a new piece of finished goods.

The following steps were used in order to fill in the buffer:

- Within MII the following transaction was created Get_AGV_WS,

- The block containing the webservice has to be displayed: CĀLLPCoWebService, 
- The new value (per example: 2080.1.1104 where 2080.1.1 is the operation number and 104 is the WT number needs to be set),

- Inside the global input variables of the transaction the following inputs are needed:

o Bst_id (per example ST2120M01BS01 (where 2120 is the operation number),

o Resources for operation 2080 the TAF129, TAF130 and TAF131 were used,

o SFC_ID is going to be changed as per test (101 was used),

o WT_ID is the AGV id.

A further programming description is presented below for the main variables used for testing purposes:

\begin{tabular}{|c|c|c|c|}
\hline production_id & String [50] 50 Byte & Read \& Write & Production ID \\
\hline motor $\mathrm{dmc}^{-} 1$ & String [50] 50 Byte & Read \& Write & Motor DMC \\
\hline motor_dmc_2 & String [50] 50 Byte & Read \& Write & Motor DMC \\
\hline achs_dmc & String [50] 50 Byte & Read \& Write & Achs DMC \\
\hline $\mathrm{vt}$ & String [50] 50 Byte & Read \& Write & AGV ID \\
\hline & UINT $\quad 2$ Byte & Read \& Write & Number Type \\
\hline tus & 1 Byte & Read \& Write & Status of AGVV \\
\hline
\end{tabular}

In order to check if the buffer was filled, a confirmation within SAP PCo has to bee displayed and checked before any test inside the destination Z_PCOBuffer_MULTIPLE_1

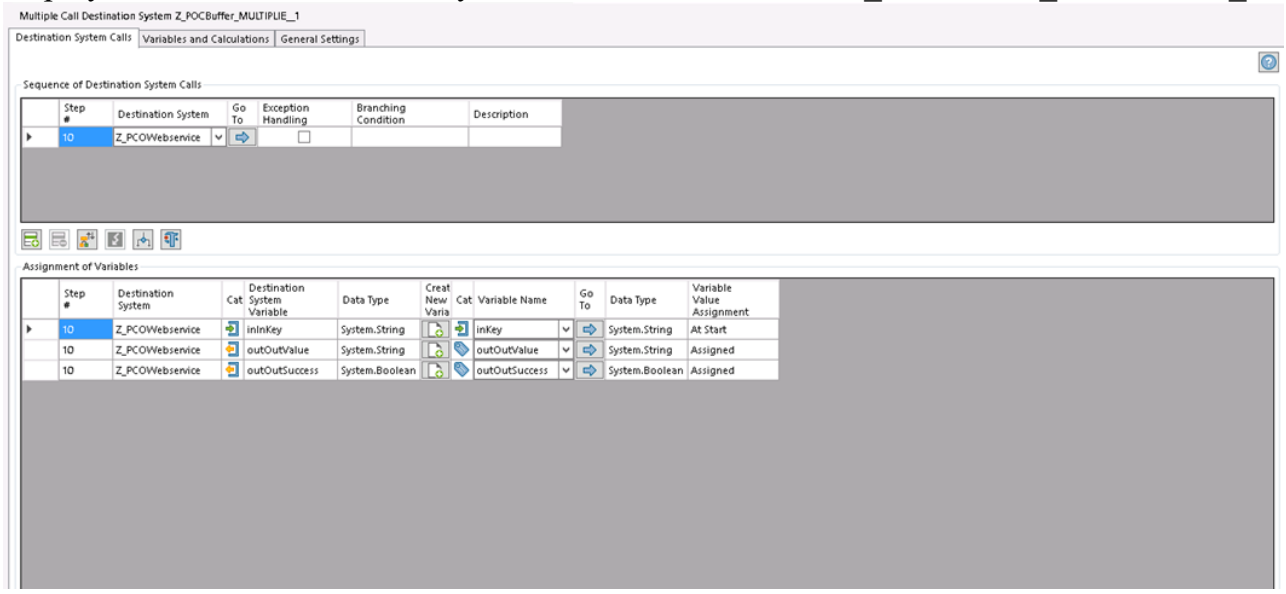

Fig. 3. Display screen for the connection between SAP PCo and SAP MII.

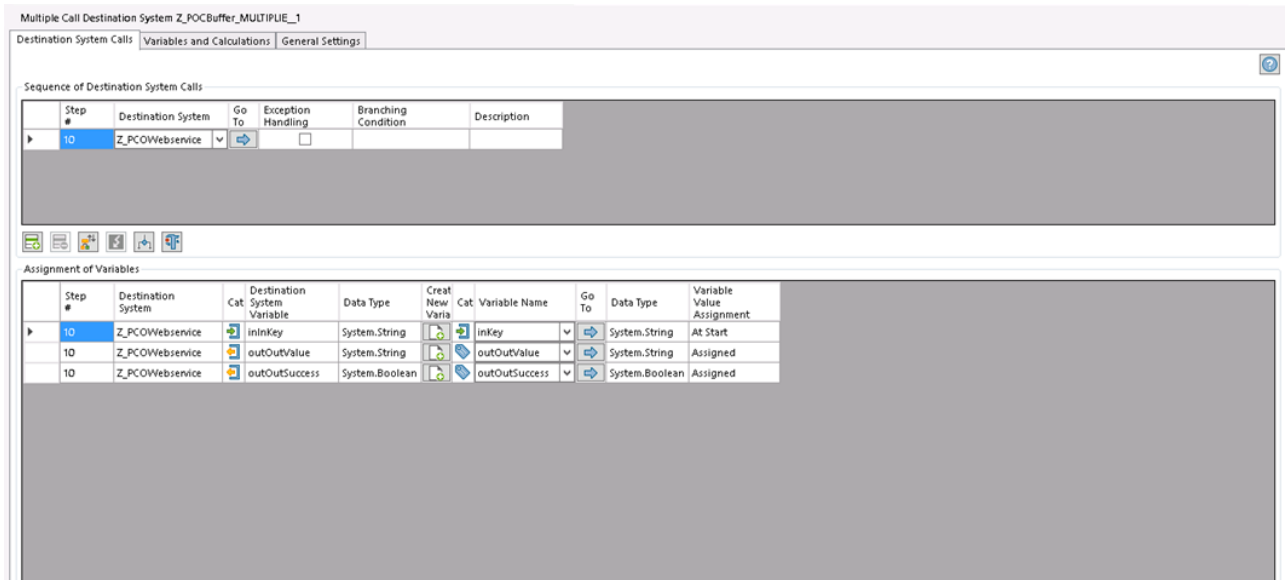

Fig. 4. Example of the buffer in SAP Plant Connectivity. 
Following the filling of the buffer and the successful and non-error test of the decision making layer, the authors have started the actual test in the live environment. The results of the test were as expected and are presented below in Fig 5. and Fig 6.

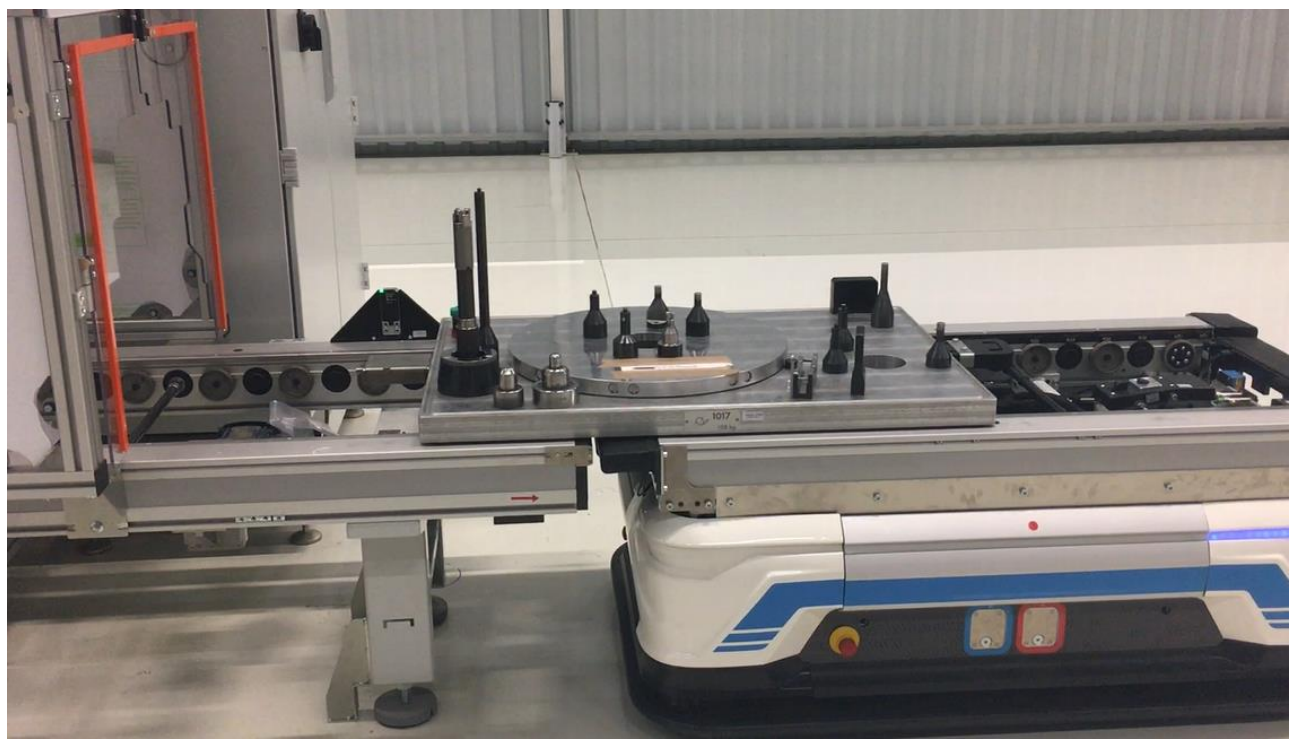

Fig. 5. The AGV represented by the white with blue stripes mobile vehicle is collecting a finished test piece from the current station.
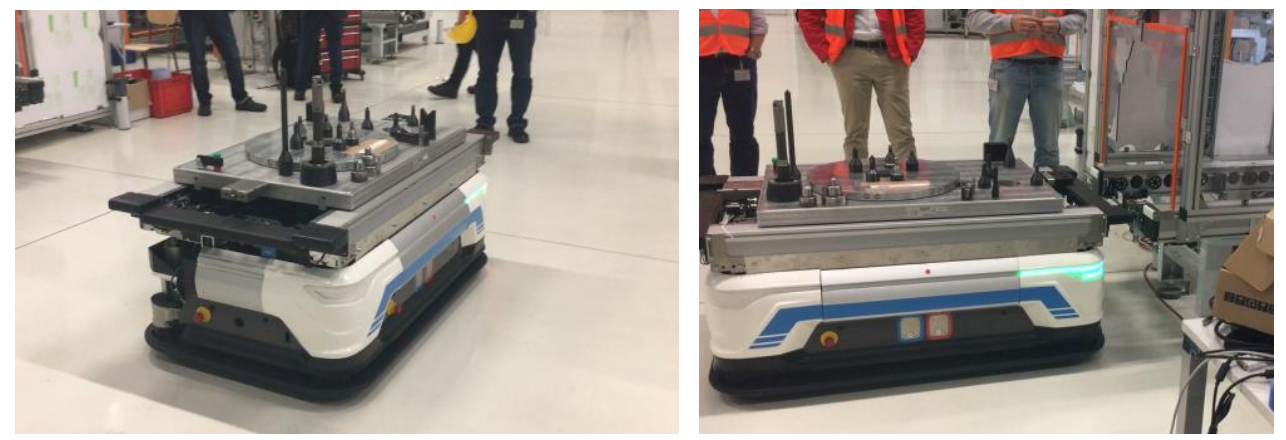

Fig. 6. Left - The AGV is transporting the finished test piece from the previous station to the next station. Right - The AGV is delivering the finished test piece to the next station.

\section{Conclusions}

Progress has been made in the field of Automated Guided Fields as per the authors have demonstrated along their testing activities. Even though the solution has been successfully implemented, gaps may rise in a live and working manufacturing plant. One of them could be the problem of autonomy, in which the AGV have not demonstrated an independence from the core system. Error minimization and improved transport time could be fixed by using more enhanced solutions in the future. From a technical perspective, the navigation system within needs more dedicated support in optimizing the predefined routes and pathways. The authors have concluded that a solution for collisions has to be further 
enhanced in the future when discussing about a manufacturing plant with many AGVs. Moreover, once the AGV technology will increase towards the end-developing saturation, the mobile vehicles can be implemented in other industries such as: commercial (luggage transportation inside airports), energy (nuclear plants inspection), medical (disposal of waste) or even in assistance to people who special need.

\section{References}

1. Punetha D., Kumar N., Vartika Mehta V., Development and Applications of Line Following Robot Based Health Care Management System, Vol. 2, Issue 8 (2013)

2. Feng, L., Koren, Y., and Borenstien, J., A Cross-Coupling Motion Controller for Mobile Robots, IEEE Journal of Control Systems, December 35-43 (1993)

3. Schulze L., Behiling S., Automated Guided Vehicle System: a Driver for Increased Business Performance, IMECS, (Hong Kong 2008)

4. M.A.Rahaman, Design And Fabrication Of Line Follower Robot, Asian journal of applied science and engineering, Vol. 2 (2013)

5. Bajestani, S.E.M., Vosoughinia, A., Technical Report of Building a Line Follower Robot, International Conference on Electronics and Information Engineering ICEIE Vol. 1, pp v1-1 v1-5 (2010)

6. Kelly A., Unnikrishnan R., A Constrained Optimization Approach to Globally Consistent Mapping, Proceedings of the IEEE/RSJ International Conference on Intelligent Robots and Systems IROS (Lausanne, Switzerland 2002)

7. Rajiv M., Atri S., Development of a Fuzzy Logic Based Mobile Robot for Dynamic Obstacle Avoidance and Goal Acquisition in an Unstructured Environment\|, International Conference on Advanced Intelligent Mechatronics, pp.235-247 (2003)

8. K Kishor, Designe Of Automated Guided Vehicle, IJARC Vol. 3, Issue 1, JanuaryApril (2012) 